Теорія Ймовір. та Матем. Статист. Вип. 76, 2007
Theor. Probability and Math. Statist.

No. 76, 2008, Pages 93-101

S 0094-9000(08)00734-5

Article electronically published on July 14, 2008

\title{
THE FIRST INTEGRALS FOR SYSTEMS OF STOCHASTIC DIFFERENTIAL EQUATIONS WITH JUMPS
}

UDC 519.21

\author{
G. L. KULINICH AND S. V. KUSHNIRENKO
}

ABSTRACT. We introduce a notion of the first integral for homogeneous stochastic differential equations. The results obtained in the paper allow us to find the first integrals for homogeneous stochastic differential equations.

\section{INTRODUCTION}

Consider a system of stochastic differential equations

$$
\begin{aligned}
& d \xi(t)= a(\xi(t)) d t+\sum_{k=1}^{n} b_{k}(\xi(t)) d w_{k}(t) \\
&+\int_{\Theta_{1}} c(\xi(t-), \theta) \tilde{\nu}(d t, d \theta)+\int_{\Theta_{2}} c(\xi(t-), \theta) \nu(d t, d \theta), \\
& \xi\left(t_{0}\right)=x_{0}, \quad t_{0} \geq 0, \quad x_{0}=\left(x_{10}, x_{20}, \ldots, x_{n 0}\right),
\end{aligned}
$$

where

$$
a(x)=\left(a_{i}(x), i=1, \ldots, n\right), \quad b_{k}(x)=\left(b_{i k}(x), i=1, \ldots, n\right),
$$

and $c(x, \theta)=\left(c_{i}(x, \theta), i=1, \ldots, n\right)$ are real nonrandom vector functions defined for $x=\left(x_{1}, \ldots, x_{n}\right) \in \mathbb{R}^{n}, \theta \in \Theta, \Theta=\Theta_{1} \cup \Theta_{2}, \Theta_{1} \cap \Theta_{2}=\varnothing ;\left(\Theta, \mathcal{B}_{\Theta}\right)$ is a measurable space; $w_{k}(t)$ are jointly independent one-dimensional Wiener processes; $\left.\nu([0, t]), A\right)$ is a Poisson measure such that $\mathrm{E} \nu([0, t], A)=t \Pi(A), A \in \mathcal{B}_{\Theta}$,

$$
\tilde{\nu}(d t, d \theta)=\nu(d t, d \theta)-\Pi(d \theta) d t,
$$

$\Pi\left(\Theta_{1}\right)=\infty, \int_{\Theta_{1}}|\theta|^{2} \Pi(d \theta)<\infty, \Pi\left(\Theta_{2}\right)<\infty$; the processes $w_{k}(t)$ and the measure $\nu([0, t], A)$ are defined on a probability space $(\Omega, \Im, \mathrm{P})$, are $\Im_{t}$-measurable for all $t \geq t_{0}$ and $A$, and are jointly independent. Here $\Im_{t} \subset \Im$ is a nondecreasing flow of $\sigma$-algebras.

Assume that the coefficients $a(x)$ and $b_{k}(x), k=1, \ldots, n$, of equation (11) are continuous functions, $\int_{\Theta_{1}} c(x, \theta) \Pi(d \theta)$ is continuous with respect to the argument $x$, and the function $c(x, \theta)$ is continuous in measure $\Pi(d \theta)$ with respect to $x$ if $\theta \in \Theta_{2}$. Further we assume that

1) there exists a constant $C>0$ such that

$$
|a(x)|^{2}+\sum_{k=1}^{n}\left|b_{k}(x)\right|^{2}+\int_{\Theta}|c(x, \theta)|^{2} \Pi(d \theta) \leq C\left[1+|x|^{2}\right]
$$

2000 Mathematics Subject Classification. Primary 60H10.

Key words and phrases. Stochastic differential equations with jumps, first integrals. 
2) for an arbitrary number $N>0$, there exists a constant $C_{N}$ such that

$$
\begin{aligned}
& \quad|a(x)-a(y)|^{2}+\sum_{k=1}^{n}\left|b_{k}(x)-b_{k}(y)\right|^{2}+\int_{\Theta_{1}}|c(x, \theta)-c(y, \theta)|^{2} \Pi(d \theta) \\
& \quad \leq C_{N}|x-y|^{2} \\
& \text { if }|x| \leq N \text { and }|y| \leq N .
\end{aligned}
$$

It is known (see [1]) that the above conditions imply the existence of a unique right continuous strong solution $\xi(t)=\left(\xi_{i}(t), i=1, \ldots, n\right)$ of equation (11).

Consider an open domain $D \subset \mathbb{R}^{n}$ such that

$$
\Pi\{\theta \in \Theta: x+c(x, \theta) \notin D\}=0 \quad \text { for all } x \in D .
$$

Let $x_{0} \in D$. Denote by $\tau_{D}\left(x_{0}\right)$ the first time when the trajectory of the solution $\xi(t)$ of equation (11) exits from the domain $D$, that is,

$$
\tau_{D}\left(x_{0}\right)=\inf \left\{t \geq t_{0}: \xi(t) \notin D\right\}
$$

if the set on the right hand side is nonempty and $\tau_{D}\left(x_{0}\right)=\infty$ otherwise.

By $\Gamma_{D}(G)$ we denote the set $\Gamma=\{x \in D: G(x)=C\}$, where $G(x)$ is twice continuously differentiable in the domain $D$, the surface $G(x)=C$ is simple in the Jordan sense, and the functions

$$
\int_{\Theta}|G(x+c(x, \theta))-G(x)|^{k} \Pi(d \theta), \quad k=1,2,
$$

are continuous in $x$.

In what follows we use the following notation: $(\cdot, \cdot)$ is the scalar product;

$$
\begin{gathered}
\nabla \cdot=\left(\frac{\partial \cdot}{\partial x_{1}}, \frac{\partial \cdot}{\partial x_{2}}, \ldots, \frac{\partial \cdot}{\partial x_{n}}\right) \\
\left(\nabla \cdot, b_{k}(x)\right)^{2} G(x)=\sum_{i=1}^{n} G_{x_{i}^{2}}^{\prime \prime}(x) b_{k i}^{2}(x)+\sum_{i, j=1, i \neq j}^{n} G_{x_{i} x_{j}}^{\prime \prime}(x) b_{k i}(x) b_{k j}(x) .
\end{gathered}
$$

Definition 1.1. A twice continuously differentiable function $G(x)$ in the domain $D$ is called a first integral of equation (1) in the domain $D$ if

$$
\left[G(\xi(t))-G\left(x_{0}\right)\right] \phi(t)=0 \quad \text { with probability } 1
$$

for all $x_{0} \in D$ and $t \geq t_{0}$, where $\phi(t)=1$ for $t_{0} \leq t<\tau_{D}\left(x_{0}\right)$ and $\phi(t)=0$ for $t \geq \tau_{D}\left(x_{0}\right)$.

Below we obtain necessary and sufficient conditions (Theorem 2.1) that a given function $G(x)$ is a first integral of equation (11) in a domain $D$. We find a relationship between first integrals of systems of homogeneous stochastic differential equations with jumps and those for some systems of ordinary differential equations. This extends some known results for the Itô stochastic differential equations (see [2]) to the case of some classes of stochastic differential equations with jumps.

\section{MAin RESUlts}

Theorem 2.1. Let $G$ be a twice continuously differentiable function in a domain $D$. Assume that $G(x)$ satisfies condition (3). The function $G$ is a first integral of equation (1) 
in the domain $D$ if and only if

$$
\begin{gathered}
\left(\nabla G(x), b_{k}(x)\right)=0, \quad k=1, \ldots, n, \\
\left(\nabla G(x), a(x)-\int_{\Theta_{1}} c(x, \theta) \Pi(d \theta)\right)+\frac{1}{2} \sum_{k=1}^{n}\left(\nabla \cdot, b_{k}(x)\right)^{2} G(x)=0, \\
\Pi\{\theta \in \Theta: G(x+c(x, \theta))-G(x) \neq 0\}=0
\end{gathered}
$$

for all $x \in D$.

Proof. Let a function $G(x)$ be twice continuously differentiable in the domain $D$, satisfy condition (3), and be a first integral of equation (10) in the domain $D$. Then the Itô formula implies for $t_{0} \leq t<\tau_{D}\left(x_{0}\right)$ that

$$
\begin{aligned}
d G(\xi(t))= & \left\{(\nabla G(\xi(t)), a(\xi(t)))+\frac{1}{2} \sum_{k=1}^{n}\left(\nabla \cdot, b_{k}(\xi(t))\right)^{2} G(\xi(t))\right. \\
& +\int_{\Theta_{1}}[G(\xi(t)+c(\xi(t), \theta))-G(\xi(t)) \\
& -(\nabla G(\xi(t)), c(\xi(t), \theta))] \Pi(d \theta)\} d t \\
& +\sum_{k=1}^{n}\left(\nabla G(\xi(t)), b_{k}(\xi(t))\right) d w_{k}(t) \\
& +\int_{\Theta_{1}}[G(\xi(t-)+c(\xi(t-), \theta))-G(\xi(t-))] \tilde{\nu}(d t, d \theta) \\
& +\int_{\Theta_{2}}[G(\xi(t-)+c(\xi(t-), \theta))-G(\xi(t-))] \nu(d t, d \theta)
\end{aligned}
$$

(see [1]) or, in other words,

$$
G(\xi(t))=G\left(x_{0}\right)+I_{1}(t)+I_{2}(t),
$$

where

$$
\begin{gathered}
I_{1}(t)=\int_{0}^{t}\left[(\nabla G(\xi(s)), a(\xi(s)))+\frac{1}{2} \sum_{k=1}^{n}\left(\nabla \cdot, b_{k}(\xi(s))\right)^{2} G(\xi(s))\right. \\
+\int_{\Theta}[G(\xi(s)+c(\xi(s), \theta))-G(\xi(s))] \Pi(d \theta) \\
\left.\quad-\int_{\Theta_{1}}[(\nabla G(\xi(s)), c(\xi(s), \theta))] \Pi(d \theta)\right] d s, \\
I_{2}(t)=\sum_{k=1}^{n} \int_{0}^{t}\left(\nabla G(\xi(s)), b_{k}(\xi(s))\right) d w_{k}(s) \\
+\int_{0}^{t} \int_{\Theta}[G(\xi(s-)+c(\xi(s-), \theta))-G(\xi(s-))] \tilde{\nu}(d s, d \theta) .
\end{gathered}
$$

Using Definition 1.1 and equality (8) we get

$$
-I_{1}(t)=I_{2}(t)
$$

for all $t_{0} \leq t<\tau_{D}\left(x_{0}\right)$.

The process $I_{1}(t)$ is absolutely continuous with probability one, that is, $I_{1}(t)$ has the derivative with probability one, while the process $I_{2}(t)$ is a local martingale, that is, $I_{2}(t)$ 
has no derivative. Thus equality (9) holds if

$$
I_{1}(t)=0, \quad I_{2}(t)=0
$$

for all $t_{0} \leq t<\tau_{D}\left(x_{0}\right)$.

Further we show that equalities (10) imply the assumptions of Theorem 2.1. Indeed, we apply the continuity of the functions (3) and the conditions imposed on the coefficients of system (1) to prove that the function

$$
\begin{aligned}
\psi(x)= & \left(\nabla G(x), a(x)-\int_{\Theta_{1}} c(x, \theta) \Pi(d \theta)\right)+\frac{1}{2} \sum_{k=1}^{n}\left(\nabla \cdot, b_{k}(x)\right)^{2} G(x) \\
& +\int_{\Theta}[G(x+c(x, \theta))-G(x)] \Pi(d \theta)
\end{aligned}
$$

is continuous.

Assume that $\psi\left(x^{0}\right) \neq 0$ at some point $x^{0} \in \Gamma_{D}(G)$. Let $\psi\left(x^{0}\right)>0$. Since the function $\psi(x)$ is continuous and the domain $D$ is open, there exists a $\delta$-neighborhood $V_{\delta}\left(x^{0}\right) \subset D$ of the point $x^{0}$ such that $\psi(x)>0$ for all $x \in V_{\delta}\left(x^{0}\right)$. Without loss of generality we assume that $x_{0}=x^{0}$ (this can be assumed in view of Definition 1.1). Denote by $\tau_{\delta}$ the first moment when the trajectory of the solution $\xi(t)$ exits from the domain $V_{\delta}\left(x_{0}\right)$.

Thus $I_{1}(t)>0$ for $t_{0} \leq t<\tau_{\delta}$. Since $\tau_{\delta} \leq \tau_{D}\left(x_{0}\right)$, we obtain a contradiction, since (10) implies that

$$
I_{1}(t)=0 \quad \text { for } t_{0} \leq t<\tau_{D}\left(x_{0}\right) .
$$

The reasoning for $\psi\left(x^{0}\right)<0$ is the same. Therefore

$$
\psi(x)=0 \quad \text { for all } x \in \Gamma_{D}(G) .
$$

Equalities (10) imply that the characteristic $\left\langle I_{2}(t)\right\rangle$ of the martingale $I_{2}(t)$,

$$
\begin{aligned}
\left\langle I_{2}(t)\right\rangle=\int_{0}^{t}\left[\sum_{k=1}^{n}(\nabla G(\xi(s)),\right. & \left.b_{k}(\xi(s))\right)^{2} \\
& \left.+\int_{\Theta}[G(\xi(s)+c(\xi(s), \theta))-G(\xi(s))]^{2} \Pi(d \theta)\right] d s
\end{aligned}
$$

is such that

$$
\left\langle I_{2}(t)\right\rangle=0
$$

for all $t_{0} \leq t<\tau_{D}\left(x_{0}\right)$.

Similarly to the proof of equality (111) we get

$$
\sum_{k=1}^{n}\left(\nabla G(x), b_{k}(x)\right)^{2}+\int_{\Theta}[G(x+c(x, \theta))-G(x)]^{2} \Pi(d \theta)=0
$$

for all $x \in \Gamma_{D}(G)$. The latter equality together with condition (11) implies equalities (4) -(6).

To prove the sufficiency we apply the condition $\Pi\left(\Theta_{2}\right)<\infty$. Thus the process $\nu\left([0, t], \Theta_{2}\right)$ has a finite number of jumps at every finite interval. The moments when the jumps occur are denoted by $\tau_{1}, \tau_{2}, \ldots$. Let $t_{0}<\tau_{1}<\tau_{2}<\cdots$ and let there exist $\theta_{k} \in \Theta_{2}$ such that the pairs $\left\{\tau_{k}, \theta_{k}\right\}$ are independent and $\nu\left(\left\{\tau_{k}\right\},\left\{\theta_{k}\right\}\right)=1$ for 
$k=1,2, \ldots$ (see [1]). Then $\xi(t)$ is a solution of the equation

$$
\begin{aligned}
\xi(t)= & \xi\left(t_{0}\right)+\int_{t_{0}}^{t} a(\xi(s)) d s+\sum_{k=1}^{n} \int_{t_{0}}^{t} b_{k}(\xi(s)) d w_{k}(s) \\
& +\int_{t_{0}}^{t} \int_{\Theta_{1}} c(\xi(s-), \theta) \tilde{\nu}(d s, d \theta)
\end{aligned}
$$

for $t_{0} \leq t<\tau_{1}$.

Let $x_{0} \in D$. Consider the curve

$$
\Gamma_{D}(G)=\left\{x \in D: G(x)=G\left(x_{0}\right)\right\} .
$$

Using the Itô formula for $t_{0} \leq t<\tau_{1}$ we get

$$
\begin{aligned}
G(\xi(t))= & G\left(x_{0}\right)+\int_{t_{0}}^{t}\left\{(\nabla G(\xi(s)), a(\xi(s)))+\frac{1}{2} \sum_{k=1}^{n}\left(\nabla \cdot, b_{k}(\xi(s))\right)^{2} G(\xi(s))\right. \\
& +\int_{\Theta_{1}}[G(\xi(s)+c(\xi(s), \theta))-G(\xi(s)) \\
& -(\nabla G(\xi(s)), c(\xi(s), \theta))] \Pi(d \theta)\} d s \\
& +\sum_{k=1}^{n} \int_{t_{0}}^{t}\left(\nabla G(\xi(s)), b_{k}(\xi(s))\right) d w_{k}(s) \\
& +\int_{t_{0}}^{t} \int_{\Theta_{1}}[G(\xi(s-)+c(\xi(s-), \theta))-G(\xi(s-))] \tilde{\nu}(d s, d \theta) .
\end{aligned}
$$

Now we derive from the assumptions of the theorem that

$$
G(\xi(t))=G\left(x_{0}\right) \quad \text { for } t_{0} \leq t<\tau_{1},
$$

that is, $\xi(t) \in \Gamma_{D}(G)$. We also have $\xi(t) \rightarrow \xi\left(\tau_{1}-0\right)$ as $t \rightarrow \tau_{1}$, that is,

$$
G(\xi(t)) \rightarrow G\left(\xi\left(\tau_{1}-0\right)\right) \quad \text { as } t \rightarrow \tau_{1}
$$

Thus

$$
G\left(\xi\left(\tau_{1}-0\right)\right)=G\left(x_{0}\right)
$$

whence $\xi\left(\tau_{1}-0\right) \in \Gamma_{D}(G)$.

By construction of the solution $\xi(t)$ of equation (1),

$$
\xi\left(\tau_{1}\right)=\xi\left(\tau_{1}-0\right)+c\left(\xi\left(\tau_{1}-0\right), \theta_{1}\right) ;
$$

hence $\xi\left(\tau_{1}\right) \in \Gamma_{D}(G)$ by the assumptions of the theorem.

Similarly, the equality

$$
\xi(t)=\xi\left(\tau_{k}\right)+\int_{\tau_{k}}^{t} a(\xi(s)) d s+\sum_{k=1}^{n} \int_{\tau_{k}}^{t} b_{k}(\xi(s)) d w_{k}(s)+\int_{\tau_{k}}^{t} \int_{\Theta_{1}} c(\xi(s-), \theta) \tilde{\nu}(d s, d \theta)
$$


holds in every interval $\tau_{k} \leq t<\tau_{k+1}$, where $k=1,2, \ldots, t<\tau_{D}\left(x_{0}\right)$, and $\xi\left(\tau_{k}\right) \in \Gamma_{D}(G)$. Using again the Itô formula for $\tau_{k} \leq t<\tau_{k+1}$, we get

$$
\begin{aligned}
G(\xi(t))= & G\left(x_{0}\right)+\int_{\tau_{k}}^{t}\left\{(\nabla G(\xi(s)), a(\xi(s)))+\frac{1}{2} \sum_{k=1}^{n}\left(\nabla \cdot, b_{k}(\xi(s))\right)^{2} G(\xi(s))\right. \\
& +\int_{\Theta_{1}}[G(\xi(s)+c(\xi(s), \theta))-G(\xi(s)) \\
& -(\nabla G(\xi(s)), c(\xi(s), \theta))] \Pi(d \theta)\} d s \\
& +\sum_{k=1}^{n} \int_{\tau_{k}}^{t}\left(\nabla G(\xi(s)), b_{k}(\xi(s))\right) d w_{k}(s) \\
& +\int_{\tau_{k}}^{t} \int_{\Theta_{1}}[G(\xi(s-)+c(\xi(s-), \theta))-G(\xi(s-))] \tilde{\nu}(d s, d \theta) .
\end{aligned}
$$

Now we deduce from the assumptions of the theorem that

$$
G(\xi(t))=G\left(\tau_{k}\right) \text { for } \tau_{k} \leq t<\tau_{k+1},
$$

that is, $\xi(t) \in \Gamma_{D}(G)$ for $\tau_{k} \leq t<\tau_{k+1}$.

Analogously $\xi\left(\tau_{k+1}-0\right)+c\left(\xi\left(\tau_{k+1}-0\right), \theta_{k+1}\right)=\xi\left(\tau_{k+1}\right)$ and $\xi\left(\tau_{k+1}\right) \in \Gamma_{D}(G)$ by the assumptions of the theorem.

Thus $G(\xi(t))=G\left(x_{0}\right)$ for $t_{0} \leq t<\tau_{D}\left(x_{0}\right)$. Since the point $x_{0} \in D$ is arbitrary, the function $G(x)$ is a first integral of equation (11) in the domain $D$ by Definition 1.1 .

Remark 2.1. Similarly to the case of systems of Itô stochastic differential equations (see [2]), first integrals of systems of stochastic differential equations with jumps can be found among first integrals of the corresponding systems of ordinary differential equations.

Corollary 2.1. Assume that the coefficients of system (1) are such that

$$
b_{k_{0}}(x) \neq 0, \quad k_{0} \in\{1, \ldots, n\},
$$

and that $G(x)$ is a first integral. Then $G(x)$ is a first integral for the following system of ordinary differential equations:

$$
\dot{x}(t)=b_{k_{0}}(x(t)) .
$$

Proof. The function $G(x)$, as a first integral of system (1), is such that

$$
\left(\nabla G(x), b_{k}(x)\right)=0, \quad k=1, \ldots, n,
$$

for all $x \in D$. This implies that condition (4) holds for all $k=k_{0}$ and $x \in D$. The latter means that $G(x)$ is a first integral of the corresponding system of ordinary differential equations in the domain $D$.

Corollary 2.2. If the coefficients of system (1) are such that $b_{k}(x)=0, k=1, \ldots, n$, then first integrals of (10) can be found among the first integrals of the following system of ordinary differential equations:

$$
\dot{x}(t)=a(x(t))-\int_{\Theta_{1}} c(x(t), \theta) \Pi(d \theta) .
$$

Proof. Indeed, if $G(x)$ is a first integral of system (1), then

$$
\left(\nabla G(x), a(x)-\int_{\Theta_{1}} c(x, \theta) \Pi(d \theta)\right)=0
$$


for all $x \in D$. The latter equality coincides with equality (5) if $b_{k}(x)=0, k=1, \ldots, n$. This means that $G(x)$ is a first integral of system (14) in the domain $D$.

Remark 2.2. Assume that a function $G(x)$ satisfies an extra condition (6) for all $x \in D$. If $G(x)$ is a first integral of system (14), then $G(x)$ is a first integral of system (11) in the domain $D$ if $b_{k}(x)=0, k=1, \ldots, n$.

Corollary 2.3. If the coefficients of system (11) are such that

$$
a(x)=0, \quad b_{k}(x)=0, \quad k=1, \ldots, n,
$$

and $\Theta_{1}=\varnothing$, then the condition

$$
\Pi\left\{\theta \in \Theta_{2}: G(x+c(x, \theta))-G(x) \neq 0\right\}=0, \quad x \in D,
$$

is necessary and sufficient for the function $G(x)$ to be a first integral of the system.

Remark 2.3. In Corollaries 2.2 and 2.3, one can drop a strong assumption that $G(x)$ is a twice continuously differentiable function. Instead, one can assume that $G(x)$ is a continuously differentiable function in the domain $D$. This is explained by the Itô formula for $G(x)$ which holds under both sets of conditions (see [1]).

Corollary 2.4. Assume that the coefficients of system (1) are such that $a(x)=0$ and $b_{k}(x)=0, k=1, \ldots, n$. If there exists a continuously differentiable function $G(x)$ such that conditions (3) hold and

$$
\begin{gathered}
\Pi\{\theta \in \Theta: G(x+c(x, \theta))-G(x) \neq 0\}=0, \\
\int_{\Theta_{1}}(\nabla G(x), c(x, \theta)) \Pi(d \theta)=0
\end{gathered}
$$

for all $x \in D$, then $G(x)$ is a first integral of system (1).

Remark 2.4. A linear function satisfies both assumptions of Corollary 2.4. A more general function $G(x)$ can also be a first integral of system (11). This is the case if a system includes a drift vector to compensate the jumps generated by the centered Poisson measure.

Example 2.1. Consider the system

$$
\begin{gathered}
d \xi(t)=\int_{\Theta_{1}} c(\xi(t-), \theta) \tilde{\nu}(d t, d \theta)+\int_{\Theta_{2}} c(\xi(t-), \theta) \nu(d t, d \theta), \\
\xi\left(t_{0}\right)=x_{0}
\end{gathered}
$$

where $c(x, \theta)=\left(\gamma_{1}(\theta) x_{1}+\gamma_{2}(\theta) x_{2},-\gamma_{2}(\theta) x_{1}+\gamma_{1}(\theta) x_{2}\right), G(x)=|x|^{2}$, and $D=\mathbb{R}^{2} \backslash(0,0)$.

Let $\Pi\left\{\theta \in \Theta:\left(1+\gamma_{1}(\theta)\right)^{2}+\gamma_{2}^{2}(\theta) \neq 1\right\}=0$. If $\Theta_{1}=\varnothing$, then the assumptions of Corollary 2.3 hold.

Thus

$$
d G(\xi(t))=\int_{\Theta_{2}}[G(\xi(t-)+c(\xi(t-), \theta))-G(\xi(t-))] \nu(d t, d \theta)=0
$$

according to system (15), that is, $G(\xi(t))=G\left(x_{0}\right)$ with probability one and the function $G(x)=|x|^{2}$ is a first integral of system (15) in the domain $D$.

If $\Theta_{1} \neq \varnothing$, then

$$
d G(\xi(t))=-2|\xi(t)|^{2} \int_{\Theta_{1}} \gamma_{1}(\theta) \Pi(d \theta) d t .
$$

Consider a drift vector defined by condition (5). In the case under consideration, condition (5) can be rewritten as follows:

$$
(\nabla G(x), a(x))=2|x|^{2} \int_{\Theta_{1}} \gamma_{1}(\theta) \Pi(d \theta) .
$$


Let, for example,

$$
a(x)=\left(x_{1} \int_{\Theta_{1}} \gamma_{1}(\theta) \Pi(d \theta), x_{2} \int_{\Theta_{1}} \gamma_{1}(\theta) \Pi(d \theta)\right),
$$

where $\gamma_{1}(\theta)=\cos \theta-1$ and $\gamma_{2}(\theta)=\sin \theta$. Then

$$
\begin{gathered}
d \xi(t)=a(\xi(t)) d t+\int_{\Theta_{1}} c(\xi(t-), \theta) \tilde{\nu}(d t, d \theta)+\int_{\Theta_{2}} c(\xi(t-), \theta) \nu(d t, d \theta), \\
\xi\left(t_{0}\right)=x_{0} .
\end{gathered}
$$

Now we obtain

$$
\begin{aligned}
d G(\xi(t))=\{(\nabla G(\xi(t)), a(\xi(t))) & \\
& \left.+\int_{\Theta_{1}}[G(\xi(t)+c(\xi(t), \theta))-G(\xi(t))-(\nabla G(\xi(t)), c(\xi(t), \theta))] \Pi(d \theta)\right\} d t \\
& +\int_{\Theta_{1}}[G(\xi(t-)+c(\xi(t-), \theta))-G(\xi(t-))] \tilde{\nu}(d t, d \theta) \\
& +\int_{\Theta_{2}}[G(\xi(t-)+c(\xi(t-), \theta))-G(\xi(t-))] \nu(d t, d \theta) \\
= & 0,
\end{aligned}
$$

that is, $G(\xi(t))=G\left(x_{0}\right)$ with probability one and the function $G(x)=|x|^{2}$ is a first integral of this system in the domain $D$.

Example 2.2. Consider the following system of ordinary differential equations:

$$
\dot{x}(t)=b_{1}(x(t)),
$$

where $b_{1}(x)=\left(x_{1}\left(x_{2}-x_{3}\right), x_{2}\left(x_{3}-x_{1}\right), x_{3}\left(x_{1}-x_{2}\right)\right)$.

This system has two first integrals $G_{1}$ and $G_{2}$ in the domain

$$
D=\left\{x: x_{1} x_{2} x_{3}\left(x_{1}-x_{3}\right) \neq 0,|x|<C\right\},
$$

namely $G_{1}(x)=x_{1}+x_{2}+x_{3}$ and $G_{2}(x)=x_{1} x_{2} x_{3}$ (see [2]). Note that $\operatorname{grad} G_{1}$ and $\operatorname{grad} G_{2}$ are linearly independent.

Consider system (1) for the case of $n=3, \Theta_{1}=\varnothing$, and $\Pi\left(\Theta_{2}\right)<\infty$.

a) Let the coefficients of system (1) be the vector $b_{1}(x)$ defined in the system of ordinary differential equations (16) and the vectors $b_{2}(x) \equiv 0, b_{3}(x) \equiv 0$,

$$
\begin{aligned}
& a_{1}(x)=\frac{1}{x_{2}\left(x_{1}-x_{3}\right)}\left[a_{2}(x) x_{1}\left(x_{3}-x_{2}\right)-x_{1} x_{2} x_{3}\left(x_{1}-x_{3}\right)^{2}\right], \\
& a_{3}(x)=\frac{1}{x_{3}\left(x_{2}-x_{1}\right)}\left[a_{2}(x) x_{1}\left(x_{3}-x_{2}\right)+x_{1} x_{2} x_{3}\left(x_{1}-x_{3}\right)^{2}\right],
\end{aligned}
$$

where $a_{2}(x)$ is an arbitrary continuously differentiable function in the domain $D$ and

$$
c(x)=\left(x_{2}-x_{1}, x_{3}-x_{2}, x_{1}-x_{3}\right) .
$$

For these coefficients, the functions $G_{1}(x)$ and $G_{2}(x)$ satisfy the assumptions of Theorem 2.1. Thus the system of stochastic differential equations (1) has two first integrals $G_{1}$ and $G_{2}$ in the domain $D$ such that grad $G_{1}$ and grad $G_{2}$ are linearly independent $\left(G_{1}\right.$ and $G_{2}$ coincide with the first integrals for the corresponding system of ordinary differential equations). A solution of the system of stochastic differential equations diffuses beyond the line of intersections of two surfaces $\left(G_{1}(x)=G_{1}\left(x_{0}\right)\right) \cap\left(G_{2}(x)=G_{2}\left(x_{0}\right)\right), x_{0} \in D$, that is, beyond the graph of the phase trajectory of a solution of the system of ordinary equations (16). 
b) Assume that the coefficients of system (1) are $b_{1}(x)$ defined in the system of ordinary equations (16) $, b_{2}(x)=(1,1,-2), b_{3}(x) \equiv 0$; and let $a(x)$ and $c(x, \theta)$ be such that $a_{1}(x)+a_{2}(x)+a_{3}(x)=0$ and $c_{1}(x, \theta)+c_{2}(x, \theta)+c_{3}(x, \theta)=0, x \in D, \theta \in \Theta_{2}$.

The function $G_{1}(x)=x_{1}+x_{2}+x_{3}$ satisfies the assumptions of Theorem 2.1 in this case, that is, $G_{1}(x)$ is a first integral of the system of stochastic differential equations (1) in the domain $D$. For other functions $c(x, \theta)$, this system of stochastic differential equations has no first integrals.

\section{Concluding Remarks}

The results obtained in the paper can be applied when constructing the mathematical models and studying the behavior of dynamical systems that evolve under random perturbations driven by stochastic processes such as "white" and "fractional" noise.

\section{BIBLIOGRAPHY}

1. I. I. Gikhman and A. V. Skorokhod, Stochastic Differential Equations and their Applications, "Naukova dumka", Kiev, 1982. (Russian) MR678374 (84j:60003)

2. G. L. Kulinich and O. V. Pereguda, Invariant Sets of Itô Stochastic Differential Equations, Kyiv University, Kyiv, 2002. (Ukrainian)

Department of General Mathematics, Faculty for Mechanics and Mathematics, National Taras Shevchenko University, Academician Glushkov Avenue 6, Kyiv 03127, Ukraine

E-mail address: bksv@univ.kiev.ua

Department of General Mathematics, Faculty for Mechanics and Mathematics, National Taras Shevchenko University, Academician Glushkov Avenue 6, Kyiv 03127, Ukraine

E-mail address: zag_mat@univ.kiev.ua

Received 12/JUL/2006

Translated by S. KVASKO 